\title{
Non - Darcian and Non - Uniform Salinity Gradients on Triple Diffusive Convection in Composite Layers
}

\author{
B. Komala, R. Sumithra
}

\begin{abstract}
The effect of uniform and non-uniform salinity gradients on the onset of triple diffusive convection in a system of composite layers enclosing an incompressible, three component, electrically conducting fluid which lies above a saturated porous layer of the identical fluid is studied analytically. The upper boundary of the fluid layer and the lower boundary of the porous layer are static and both the boundaries are insulating to heat and mass. At the interface, the velocity, shear stress, normal stress, heat, heat flux, mass and mass flux are presumed to be continuous, intended for Darcy-Brinkman model. An Eigenvalue problem is attained and the same is solved by the regular perturbation approach. The critical Rayleigh number which is the guiding principle for the invariability of the system is accomplished for every salinity profile individually. The effects of various physical parameters on the onset of Triple diffusive convection are considered for all the profiles graphically.
\end{abstract}

Keywords: Triple diffusion, non-uniform Salinity gradients, Regular perturbation method, Darcy-Brinkman model.

\section{INTRODUCTION}

In standard Benard problem, density difference was the only destabilizing source due to which the system was unstable. This unstability is due to the difference in temperature between the two surface boundaries of the fluid. This situation where the temperature is the only diffusing component is referred to as single component diffusion. If the fluid has additional salt dissolved in it then there are two destabilizing sources for the density difference i.e. temperature field and salt field, which is known as double diffusion. Along with the temperature, if there are two more agencies (salts) present dissolved in the fluid the convection is referred to as triple diffusive convection. The effect of a third diffusive agent is receiving muchattention in present day research field asthere are numerous physical systems withtwo dissolved salts diffusing independentlyalong with temperature field.

Revised Manuscript Received on July 22, 2019.

B. Komala, Research Scholar, Research and Development Centre, Bharathiar University, Coimbatore - 641046

Tamil nadu, INDIA.

R. Sumithra, Associate Professor, Department of Mathematics, Government Science College, Bangalore - 560001, Karnataka, INDIA

Griffiths [4], Turner [19] recognized that there are many situations where more than two dissolved salts are present along with the temperature field. For instance: solidification of molten alloys, geothermally heated lakes, oceanography, high-quality crystal production, oceanography,production of pure medication, undergroundwater flow and many more.

Griffiths [4], Pearlstein et al [8] and Lopez [5] investigated theoretically the onset of convection in an infinite horizontal layer of triple diffusive fluid. Shivakumara I S and Kumar [16] investigated the bifurcation analysis of a triply diffusive coupled stress fluid in terms of a simplified model consisting of seven nonlinear ordinary differential equations. Shivakumara I S and Kumar [17] have studied the linear and weaklynonlinear triple diffusive convection in couple stress fluid layer. K.R. Raghunathaand I.S Shivakumara [9] have investigatedthe triple diffusive convection in an Oldroyd-B fluidsaturated porous layer by performing linear and weakly nonlinear stabilityanalyses. Sameena Tarannum and S. Pranesh [14] have studied a nonlinear triple diffusive convection in a rotating couple stress liquid to study the effect of heat and mass transfer by deriving Ginzburg Landau equation. Chand $S$ [1] studied theoretically the triple-diffusive convection in a micropolar ferrofluid layer heated and soluted below with transverse uniform magnetic field along with uniform vertical rotation. Rana G.C et al [11] have studied the onset of triplediffusive convection in a horizontal layer of nano fluid heated from below and salted from above and below both analytically and numerically. Rionero [12] studied a triply convective diffusive fluid mixture saturating a porous horizontal layer, heated from below and salted from above. Rionero [13] also investigated the multicomponent diffusive convection in the porous layer for the more general case when heated from below and salted by $m$ salts partly from above and partly from below. Zhao, Wang and Zhang [22] investigated the problem of triply diffusive convection in a Maxwell fluid saturated porous layer. K.R. Raghunath et al [10] investigated the weakly nonlinear stability of 
the triple diffusive convection in a Maxwell fluid saturated porous layer. Mukesh Kumar Awasthi et al [6] have performed a linear stability analysis for the onset of triple-diffusive convection in the presence of internal heat source in a Maxwell fluid saturated porous layer.

All the above literature are confined to the single layer of fluid or porous layer but in many physical systems, the occurrence of composite layer and salinity gradients is natural which motivated us to study the onset of triple diffusive convection in fluid - porous composite layer for uniform and non-uniform salinity gradients.

For Fluid layer,

$$
\begin{aligned}
& \nabla \cdot \stackrel{\mathrm{I}}{q}=0 \\
& \hline \rho_{0}\left[\frac{\partial \stackrel{\mathrm{r}}{q}}{\partial t}+(\stackrel{\mathrm{r}}{q} \cdot \nabla) \stackrel{\mathrm{r}}{q}\right]=-\nabla P+\mu \nabla^{2} \stackrel{\mathrm{r}}{q}-\rho g \hat{k} \\
& \hline \frac{\partial T}{\partial t}+(\stackrel{\mathrm{r}}{q} \cdot \nabla) T=\kappa \nabla^{2} T
\end{aligned}
$$

\section{FORMULATION OF THE PROBLEM}

We consider a horizontal three component, electrically conducting fluid saturated isotropic sparsely packed porous layer of thickness $d_{m}$ underlying a three component fluid layer of thickness $d$. The lower surface of the porous layer and the upper surface of the fluid layer are bounded by rigid walls. Both the boundaries are kept at different constant temperatures and salinities. A Cartesian coordinate system is chosen with the origin at the interface between porous and fluid layers and the $\mathrm{z}$ - axis vertically upwards.

The governing equations are continuity equation, momentum equation, energy equation, species concentration equations, and the equation of state are as follows,

$$
\frac{\left|\frac{\partial C_{1}}{\partial t}+(\stackrel{\mathrm{r}}{q} \cdot \nabla) C_{1}=\kappa_{1} \nabla^{2} C_{1}\right|}{\frac{\partial C_{2}}{\partial t}+(\stackrel{\mathrm{r}}{q} \cdot \nabla) C_{2}=\kappa_{2} \nabla^{2} C_{2}}
$$

where

$$
\left.\rho=\rho_{0}\left[1-\alpha_{t}\left(T-T_{0}\right)+\alpha_{s 1}\left(C_{1}-C_{0}\right)+\alpha_{s 2}\left(C_{2}-C_{0}\right)\right]\right\rfloor
$$

and for the porous layer,

$$
\mid \begin{aligned}
& \left|\nabla_{m} \cdot \stackrel{\mathbf{l}}{q}_{m}=0\right| \\
& \hline \rho_{0}\left[\frac{1}{\varepsilon} \frac{\partial \stackrel{\mathrm{q}}{m}_{m}}{\partial t}+\frac{1}{\varepsilon^{2}}\left(\stackrel{\mathrm{r}}{q_{m}} \cdot \nabla_{m}\right) \stackrel{\mathrm{r}}{q_{m}}\right]=-\nabla_{m} P_{m}+\mu \nabla^{2} \stackrel{\mathrm{r}}{q}_{m}-\frac{\mu}{K} \stackrel{\mathrm{r}}{q}_{m}-\rho_{m} g \hat{k}
\end{aligned}
$$

$$
\begin{array}{|l}
A \frac{\partial T_{m}}{\partial t}+\left(\stackrel{\mathrm{r}}{q}_{m} \cdot \nabla_{m}\right) T_{m}=\kappa_{m} \nabla_{m}^{2} T_{m} \\
\phi \frac{\partial C_{m 1}}{\partial t}+\left(\stackrel{\mathrm{r}}{q}_{m} \cdot \nabla_{m}\right) C_{m 1}=\kappa_{m 1} \nabla_{m}^{2} C_{m 1} \\
\phi \frac{\partial C_{m 2}}{\partial t}+\left(\stackrel{\mathrm{r}}{q}_{m} \cdot \nabla_{m}\right) C_{m 2}=\kappa_{m 2} \nabla_{m}^{2} C_{m 2}
\end{array}
$$

where

$$
\rho_{m}=\rho_{0}\left[1-\alpha_{t m}\left(T_{m}-T_{0}\right)+\alpha_{s m 1}\left(C_{m 1}-C_{0}\right)+\alpha_{s m 2}\left(C_{m 2}-C_{0}\right)\right\rfloor
$$

and the symbols in the above equations have the following meaning

$|\stackrel{r}{q}=(u, v, w)|$ is the velocity vector, $t$ is the time, $\mu$ is the fluid viscosity, $P$ is the total pressure, $\rho_{0}$ is the fluid density, $\begin{aligned} & l \\ & g\end{aligned}$ is the acceleration due to the gravity,
$A=\frac{\left(\rho_{0} C_{p}\right)_{m}}{\left(\rho C_{p}\right)_{f}}$ is the ratio of heat capacities, $C_{p}$ is the specific heat, $K$ is the permeability of the porous medium, $T$ is the temperature, $K$ is the thermal diffusivity of the fluid, 
$\left\lfloor C_{1}, C_{2}\right\rfloor$ are the concentrations or the salinity fields, $\left\lfloor\kappa_{m}\right.$

is the solute diffusivity of the fluid,

$\alpha_{t}=-\frac{1}{\rho}\left(\frac{\partial \rho}{\partial T}\right)_{P, T}, \alpha_{s 1}=-\frac{1}{\rho}\left(\frac{\partial \rho}{\partial C}\right)_{P, C_{1}}, \alpha_{s 2}=-\frac{1}{\rho}\left(\frac{\partial \rho}{\partial C}\right)_{P, C_{2}} \phi$ is the porosity and the subscripts $m$ and $f$ refer to the porous medium and the fluid respectively.

The basic steady state is assumed to the quiescent and we consider the solution of the form,

In the fluid layer,

$\left[u, v, w, P, T, C_{1}, C_{2}\right]=\left[0,0,0, P_{b}(z), T_{b}(z), C_{b 1}(z), C_{b 2}(z)\right]$

and in the porous layer

$\left[u_{m}, v_{m}, w_{m}, P_{m}, T_{m}, C_{m 1}, C_{m 2}\right]=\left[0,0,0, P_{m b}\left(z_{m}\right), T_{m b}\left(z_{m}\right), C_{m b 1}\left(z_{m}\right), C_{m b 2}\left(z_{m}\right)\right]$

where

the subscript $\left[b^{\prime}\right]$ denotes the basic state.

The temperature distributions $\left\lfloor T_{b}(z),\left|T_{m b}\left(z_{m}\right)\right|\right.$ are found to be

$$
\begin{aligned}
& T_{b}(z)=T_{0}+\frac{\left(T_{u}-T_{0}\right) z}{d} \text { in } 0 \leq z \leq d \\
& T_{m b}\left(z_{m}\right)=T_{0}-\frac{\left(T_{l}-T_{0}\right) z_{m}}{d_{m}} \text { in } 0 \leq z_{m} \leq d_{m} \\
& T_{0}=\frac{\kappa d_{m} T_{u}+\kappa_{m} d T_{l}}{\kappa d_{m}+\kappa_{m} d} \text { is the interface temperature. }
\end{aligned}
$$

The concentration distributions $C_{b 1}(z), C_{m b 1}\left(z_{m}\right), C_{b 2}(z)$ and $C_{m b 2}\left(z_{m}\right)$, are found to be

$$
\begin{array}{|l}
-\frac{\partial C_{b 1}}{\partial z}=\frac{C_{10}-C_{1 u}}{d} h(z) \mid \text { in } 0 \leq z \leq d \\
-\frac{\partial C_{m b 1}}{\partial z_{m}}=\frac{C_{1 L}-C_{10}}{d_{m}} h_{m}\left(z_{m}\right) \text { in } 0 \leq z_{m} \leq d_{m}
\end{array}
$$

(18)

$C_{b 2}(z)=C_{20}+\frac{\left(C_{2 u}-C_{20}\right) z}{d}$ in $0 \leq z \leq d$

$C_{m b 2}\left(z_{m}\right)=C_{20}-\frac{\left(C_{2 l}-C_{20}\right) z_{m}}{d_{m}}$ in $0 \leq z_{m} \leq d_{m}$

$h(z), \quad h_{m}\left(z_{m}\right)$ are salinity gradients in fluid and porous layers respectively At the interface $h(z)=h_{m}\left(z_{m}\right)$ and $C_{0}=\frac{\kappa_{s} d_{m} C_{u}+\kappa_{s m} d C_{l}}{\kappa_{s} d_{m}+\kappa_{s m} d} \quad$ is concentration at the interface.

In order to investigate the stability of the basic solution, infinitesimal disturbances are introduced in the form,

$\left[\stackrel{\mathrm{r}}{q}, P, T, C_{1}, C_{2}\right]=\left[0, P_{b}(z), T_{b}(z), C_{b 1}(z), C_{b 2}(z)\right]+\left[\stackrel{\mathrm{r}}{q^{\prime}}, P^{\prime}, \theta, S_{1}, S_{2}\right]$

and

$\left[\stackrel{\mathrm{r}}{q}_{m}, P_{m}, T_{m}, C_{m 1}, C_{m 2}\right]=\left[0, P_{m b}\left(z_{m}\right), T_{m b}\left(z_{m}\right), C_{m b 1}\left(z_{m}\right), C_{m b 2}\left(z_{m}\right)\right]+\left[\stackrel{\mathrm{r}}{q_{m}^{\prime}}, P_{m}^{\prime}, \theta_{m}, S_{m 1}, S_{m 2}\right]$ 


\section{Non - Darcian and Non - Uniform Salinity Gradients on Triple Diffusive Convection in Composite Layers}

The primed quantities in the above equations are the perturbed ones over their equilibrium counterparts. Eqs.(21) and (22) are substituted into the Eqs.(1) to (12) and are linearized in the usual manner, the pressure term is eliminated from (2) and (8) by taking curl twice on

$$
\left\lfloor(x, y, z)=d\left(x^{\prime}, y^{\prime}, z^{\prime}\right)\right\rfloor \text { and }\left\lfloor\left(x_{m}, y_{m}, z_{m}\right)=d_{m}\left(x_{m}^{\prime}, y_{m}^{\prime}, z_{m}^{\prime}-1\right)\right\rfloor
$$

In this manner the detailed flow fields in both the fluid and porous layers can be clearly obtained for all the depth ratios $\hat{d}=\frac{d}{d}$. The non dimensionalised basic equations are subjected to normal mode expansion and we seek solutions for the dependent variables in the fluid and porous layers (following Venkatachalappa $M$ et al [20]). Assuming that the principle of exchange of instabilities holds for the superposed layers (following In $0 \leq z \leq 1$ these two equations and only the vertical component is retained. The separate length scales are chosen for the two layers (following Chen and Chen [2], D.A Nield [7]), so that each layer is of unit depth with

$\left(D^{2}-a^{2}\right)^{2} W=R a^{2} \Theta-R_{s 1} a^{2} \Sigma_{1}-R_{s 2} a^{2} \Sigma_{2}$
$\left(D^{2}-a^{2}\right) \Theta+W=0$
$\tau_{1}\left(D^{2}-a^{2}\right) \Sigma_{1}+W h(z)=0$
$\tau_{2}\left(D^{2}-a^{2}\right) \Sigma_{2}+W=0$

In $0 \leq z_{m} \leq 1$

$$
\begin{aligned}
& {\left[\left(D_{m}^{2}-a_{m}^{2}\right) \hat{\mu} \beta^{2}-1\right]\left(D_{m}^{2}-a_{m}^{2}\right) W_{m}=R_{m} a_{m}^{2} \Theta_{m}-R_{s m 1} a_{m}^{2} \Sigma_{m 1}-R_{s m 2} a_{m}^{2} \Sigma_{m 2}} \\
& \left(D_{m}^{2}-a_{m}^{2}\right) \Theta_{m}+W_{m}=0 \\
& \tau_{p m 1}\left(D_{m}^{2}-a_{m}^{2}\right) \Sigma_{m 1}+W_{m} h_{m}\left(z_{m}\right)=0 \\
& \tau_{p m 2}\left(D_{m}^{2}-a_{m}^{2}\right) \Sigma_{m 2}+W_{m}=0 \\
&
\end{aligned}
$$

For the fluid layer, $\left|R=\frac{g \alpha_{t}\left(T_{0}-T_{u}\right) d^{3}}{v \kappa}\right|$ is the Rayleigh number, $\quad R_{s 1}=\frac{g \alpha_{s 1}\left(C_{10}-C_{1 u}\right) d^{3}}{v \kappa} \mid$,

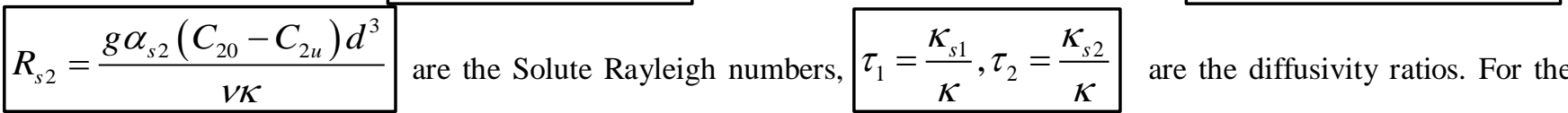
porous layer, $\beta^{2}=\frac{K}{d_{m}^{2}}=D a$ is the Darcy number, $\hat{\mu}=\frac{v_{m}}{v}$ is the viscosity ratio $R_{m}=\frac{g \alpha_{t}\left(T_{0}-T_{u}\right) d_{m} K}{v \kappa_{m}}=R D a$ is the Rayleigh - Darcy number $R_{s m 1}=\frac{g \alpha_{s 1}\left(C_{1 l}-C_{10}\right) d_{m} K}{v \kappa_{m}}=R_{s 1} D a, R_{s m 2}=\frac{g \alpha_{s 2}\left(C_{2 l}-C_{20}\right) d_{m} K}{v \kappa_{m}}=R_{s 2} D a$ are the Solute Rayleigh - Darcy number in porous medium, $\tau_{p m 1}=\frac{\kappa_{s m 1}}{\kappa_{m}}, \tau_{p m 2}=\frac{\kappa_{s m 2}}{\kappa_{m}}$ are the diffusivity ratios, $a$ and $a_{m}$ are the non-dimensional horizontal wave numbers,,$\theta$ and $\theta_{m}$ are the temperature in fluid and porous layers, $S$ and $S_{m}$ are the concentration in fluid and porous layers and $\mid \int_{0}^{1} h(z) d z=\int_{0}^{1} h_{m}\left(z_{m}\right) d z_{m}=1$.

Eqns. (23) to (30) are twentieth order ordinary differential equation which are to be solved using the below mentioned boundary conditions. 


\section{BOUNDARY CONDITIONS}

The boundary conditions after non-dimensionalisation and Normal mode expansion are

\begin{tabular}{l}
$\mid W(1)=0, D W(1)=0, D \Theta(1)=0, \quad D S_{1}(1)=0, D S_{2}(1)=0, \quad D_{m} S_{m 1}(0)=0, \quad D_{m} S_{m 2}(0)=0$, \\
\hline$\hat{T} W(0)=W_{m}(1), \hat{T} \hat{d} D W(0)=D_{m} W_{m}(1), \quad\left[\hat{T} \hat{d}^{2}\left(D^{2}+a^{2}\right) W(0)=\hat{\mu}\left(D_{m}^{2}+a_{m}^{2}\right) W_{m}(1)\right.$ \\
$\Theta(0)=\hat{T} \Theta_{m}(1), \quad D \Theta(0)=D_{m} \Theta_{m}(1), S_{1}(0)=\hat{S} S_{m 1}(1), \quad D S_{1}(0)=D_{m} S_{m 1}(1)$ \\
\hline \hline$S_{2}(0)=\hat{S} S_{m 2}(1), \quad D S_{2}(0)=D_{m} S_{m 2}(1), \quad W_{m}(0)=0, \quad D_{m} W_{m}(0)=0, \quad D_{m} \Theta_{m}(0)=0$, \\
\hline$\hat{T} \hat{d}^{2} \beta^{2}\left(D^{3} W(0)-3 a^{2} D W(0)\right)=-D_{m} W_{m}(1)+\hat{\mu} \beta^{2}\left(D_{m}^{3} W_{m}(1)-3 a_{m}^{2} D_{m} W_{m}(1)\right)$
\end{tabular}

where

$$
\hat{T}=\left(T_{l}-T_{0}\right) /\left(T_{0}-T_{u}\right), \quad \hat{\kappa}_{s}=\kappa_{s m} / \kappa_{s}=\hat{d} / \hat{S}, \quad \hat{S}_{i}=\left(C_{i l}-C_{i 0}\right) /\left(C_{i 0}-C_{i u}\right)
$$

$\hat{\kappa}=\kappa_{m} / \kappa=\hat{d} / \hat{T}, \quad \hat{\kappa}_{s 1}=\kappa_{s m 1} / \kappa_{s 1}=\hat{d} / \hat{S}_{1}$ and $\hat{\kappa}_{s 2}=\kappa_{s m 2} / \kappa_{s 2}=\hat{d} / \hat{S}_{2} \cdot \hat{\kappa}, \quad \hat{\kappa}_{s 1}$ and

thermal diffusivity and the solutal diffusivity ratios respectively. The Energy Equations are solved using respective boundary conditions from (29) (following Shivakumara I.S et al [15]).

\section{SOLUTION BY REGULAR PERTURBATION TECHNIQUE}

For the constant heat and mass flux boundaries convection sets in at small values of horizontal wavenumber 'a', accordingly, we expand

$\left[\begin{array}{c}W \\ \Theta \\ \Sigma_{1} \\ \Sigma_{2}\end{array}\right]=\sum_{j=0}^{\infty} a^{2 j}\left[\begin{array}{c}W_{j} \\ \Theta_{j} \\ \Sigma_{j 1} \\ \Sigma_{j 2}\end{array}\right]$ and $\left[\begin{array}{c}W_{m} \\ \Theta_{m} \\ \Sigma_{m 1} \\ \Sigma_{m 2}\end{array}\right]=\sum_{j=0}^{\infty} a^{2 j}\left[\begin{array}{c}W_{m j} \\ \Theta_{m j} \\ \Sigma_{m j 1} \\ \Sigma_{m j 2}\end{array}\right]$

With an arbitrary factor, the solutions for zero order equations are:

$$
\mid \begin{aligned}
& W_{0}(z)=0, \quad \Theta_{0}(z)=\hat{T}, \quad \Sigma_{10}(z)=\hat{S}_{1}, \quad \Sigma_{20}(z)=\hat{S}_{2} \\
& W_{m 0}\left(z_{m}\right)=0, \quad \Theta_{m 0}\left(z_{m}\right)=1, \quad \Sigma_{m 10}\left(z_{m}\right)=1, \quad \Sigma_{m 20}\left(z_{m}\right)=1
\end{aligned}
$$

The equations at first order in $\left\lfloor a^{2}\right\rfloor$ are,

For fluid layer,

$$
\begin{aligned}
& D^{4} W_{1}-R \hat{T}+R_{s 1} \hat{S}_{1}+R_{s 2} \hat{S}_{2}=0 \\
& D^{2} \Theta_{1}-\hat{T}+W_{1}=0 \\
& \hline \tau_{1} D^{2} \Sigma_{11}-\tau_{1} \hat{S}_{1}+W_{1} h(z)=0 \\
& \tau_{2} D^{2} \Sigma_{21}-\tau_{2} \hat{S}_{2}+W_{1}=0
\end{aligned}
$$

For porous layer,

$$
\begin{gathered}
\mid \hat{\mu} \beta^{2} D_{m}^{4} W_{m 1}-D_{m}^{2} W_{m 1}-R_{m}+R_{s m 1}+R_{s m 2}=0 \\
\hline D_{m}^{2} \Theta_{m 1}-1+W_{m 1}=0 \\
\hline \tau_{m 1} D_{m}^{2} \Sigma_{m 1}-\tau_{m 1}+W_{m 1} h_{m}\left(z_{m}\right)=0 \\
\frac{\tau_{m 2} D_{m}^{2} \Sigma_{m 2}-\tau_{m 2}+W_{m 1} h_{m}\left(z_{m}\right)=0}{(38)}
\end{gathered}
$$

The corresponding boundary conditions are, 


$$
\begin{aligned}
& \begin{array}{l}
\mid W_{1}(1)=0, D W_{1}(1)=0, D \Theta_{1}(1)=0, D S_{1}(1)=0 \\
\hat{T} \hat{d} D W_{1}(0)=\hat{d}^{2} D_{m} W_{m 1}(1), \hat{T} \hat{d}^{2} D^{2} W_{1}(0)=\hat{\mu} D_{m}^{2} W_{m 1}(1) \hat{d}^{2}
\end{array}
\end{aligned}
$$

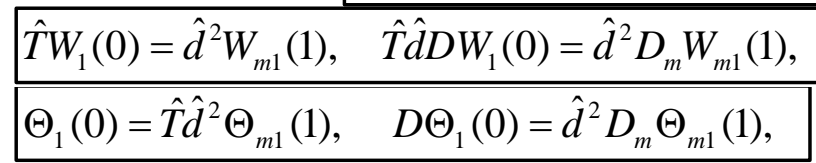

$$
\begin{aligned}
& S_{1}(0)=\hat{S}_{1} \hat{d}^{2} S_{m 1}(1), \quad D S_{1}(0)=\hat{d}^{2} D_{m} S_{m 1}(1), \quad S_{2}(0)=\hat{S}_{1} \hat{d}^{2} S_{m 2}(1), \\
& \begin{array}{l}
\hat{T} \hat{d}^{3} \beta^{2} D^{3} W_{1}(0)=-\hat{d}^{2} D_{m} W_{m 1}(1)+\hat{\mu} \beta^{2} \hat{d}^{2} D_{m}^{3} W_{m 1}(1), \quad D S_{2}(0)=\hat{d}^{2} D_{m} S_{m 2}(1), \\
W_{m 1}(0)=0, D_{m} W_{m 1}(0)=0, D_{m} \Theta_{m 1}(0)=0, D_{m} S_{m 1}(0)=0, D_{m} S_{m 2}(0)=0
\end{array}
\end{aligned}
$$

The solutions of the Eqs.(32) and (36) give $W_{1}$ and $W_{m 1}$ respectively are important in obtaining the Eigen values and are found to be,

$$
\begin{aligned}
& \left\lfloor W_{1}(z)=C_{1}+C_{2} z+C_{3} z^{2}+C_{4} z^{3}+\left(R \hat{T}-R_{s 1} \hat{S}_{1}-R_{s 2} \hat{S}_{2}\right) \frac{z^{4}}{24} \mid\right. \\
& \left.W_{m 1}\left(z_{m}\right)=C_{5}+C_{6} z_{m}+C_{7} e^{p z_{m}}+C_{7} e^{-p z_{m}}-\left(R_{m}-R_{s m 1}-R_{s m 2}\right) \frac{z_{m}^{2}}{2}\right\rfloor
\end{aligned}
$$

\begin{tabular}{|c|c|}
\hline$\Delta_{3}=\frac{\hat{\mu} p^{2} e^{p}}{2 \hat{T}}, \quad \Delta_{4}=\frac{\hat{\mu} p^{2} e^{-p}}{2 \hat{T}}, \quad \Delta_{5}=\frac{\hat{d}}{\hat{T}}\left(p e^{p}-p\right), \quad \Delta_{6}=\frac{\hat{d}}{\hat{T}}\left(p-p e^{-p}\right), \quad \Delta_{7}$ & $=\frac{\hat{d}^{2}}{\hat{T}}\left(e^{p}-p-1\right)$ \\
\hline$\Delta_{8}=\frac{\hat{d}^{2}}{\hat{T}}\left(e^{-p}-p-1\right), \quad \Delta_{9}=\Delta_{7}+\Delta_{5}+\Delta_{3}+\Delta_{1}, \quad \Delta_{10}=\Delta_{8}+\Delta_{6}+\Delta_{4}+\Delta_{2}$ & \\
\hline$\Delta_{11}=\frac{1}{6 \hat{T} \hat{d} \beta^{2}}-\frac{\hat{\mu}}{2 \hat{T}}-\frac{\hat{d}}{\hat{T}}-\frac{\hat{d}^{2}}{2 \hat{T}}, \quad \Delta_{12}=\Delta_{5}+2 \Delta_{3}+3 \Delta_{1}, \quad \Delta_{13}=\Delta_{6}+2 \Delta_{4}+3 \Delta_{2}$, & \\
\hline \begin{tabular}{|l}
$\Delta_{14}=\frac{1}{2 \hat{T} \hat{d} \beta^{2}}-\frac{\hat{d}}{\hat{T}}-\frac{\hat{\mu}}{\hat{T}}, \quad \Delta_{15}=\frac{\frac{\Delta_{9}}{6}-\frac{\Delta_{12}}{24}}{\Delta_{10} \Delta_{12}-\Delta_{9} \Delta_{13}}, \quad \Delta_{16}=\frac{\Delta_{9} \Delta_{14}-\Delta_{11} \Delta_{12}}{\Delta_{10} \Delta_{12}-\Delta_{9} \Delta_{13}}$ \\
$\Delta_{17}=-\left(\frac{\Delta_{10} \Delta_{15}}{\Delta_{9}}+\frac{1}{24 \Delta_{9}}\right), \quad \Delta_{18}=-\left(\frac{\Delta_{10} \Delta_{16}+\Delta_{11}}{\Delta_{9}}\right), \quad \Delta_{19}=\Delta_{7} \Delta_{17}+\Delta_{15} \Delta_{8}$
\end{tabular} & \\
\hline
\end{tabular}

Where $p=\sqrt{\frac{1}{\hat{\mu} \beta^{2}}}$ and $C_{1}, C_{2}, C_{3}, C_{4}, C_{5}, C_{6}, C_{7}, C_{8}$ are constants which are determined using the velocity boundary conditions and are as follows

$$
\left\{\begin{array}{l}
C_{1}=\Delta_{7} C_{7}+\Delta_{8} C_{8}-\frac{\hat{d}^{2} B}{2 \hat{T}}, \quad C_{2}=\Delta_{5} C_{7}+\Delta_{6} C_{8}-\frac{\hat{d}^{2} B}{\hat{T}}, \quad C_{3}=\Delta_{3} C_{7}+\Delta_{4} C_{8}-\frac{\hat{\mu} B}{2 \hat{T}}, \\
C_{4}=\Delta_{1} C_{7}+\Delta_{2} C_{8}+\frac{B}{6 \hat{T} \hat{d} \beta^{2}}, \quad C_{5}=-C_{7}-C_{8}, \quad C_{6}=p C_{8}-p C_{7}, \quad C_{7}=A \Delta_{17}+B \Delta_{18}, \\
C_{8}=A \Delta_{15}+B \Delta_{16}, \quad A=R \hat{T}-R_{s 1} \hat{S}_{1}-R_{s 2} \hat{S}_{2}, \quad B=R_{m}-R_{s m 1}-R_{s m 2}, \\
\hline \Delta_{1}=\frac{\hat{\mu} \beta^{2} p^{3} e^{p}-p e^{p}+p}{6 \hat{T} \hat{d} \beta^{2}}, \quad \Delta_{2}=\frac{p e^{-p}-p-\hat{\mu} \beta^{2} p^{3} e^{-p}}{6 \hat{T} \hat{d} \beta^{2}}
\end{array}\right.
$$




$$
\left\{\begin{array}{l}
\Delta_{20}=\Delta_{7} \Delta_{18}+\Delta_{16} \Delta_{8}-\frac{\hat{d}^{2}}{2 \hat{T}}, \quad \Delta_{21}=\Delta_{5} \Delta_{17}+\Delta_{15} \Delta_{6}, \quad \Delta_{22}=\Delta_{5} \Delta_{18}+\Delta_{16} \Delta_{6}-\frac{\hat{d}}{\hat{T}}, \quad \Delta_{28}=-\Delta_{18}-\Delta_{16}, \\
\Delta_{23}=\Delta_{3} \Delta_{17}+\Delta_{15} \Delta_{4}, \quad \Delta_{24}=\Delta_{3} \Delta_{18}+\Delta_{16} \Delta_{4}-\frac{\hat{\mu}}{2 \hat{T}}, \quad \Delta_{25}=\Delta_{1} \Delta_{17}+\Delta_{15} \Delta_{2}, \quad \Delta_{30}=p\left(\Delta_{16}-\Delta_{18}\right) \\
\Delta_{26}=\Delta_{1} \Delta_{18}+\Delta_{16} \Delta_{2}+\frac{1}{6 \hat{T} \hat{d} \beta^{2}}, \quad \Delta_{27}=-\Delta_{17}-\Delta_{15}, \quad \Delta_{29}=p\left(\Delta_{15}-\Delta_{17}\right) .
\end{array}\right.
$$

\subsection{Solvability condition}

The differential equations and boundary conditions corresponding to temperature and concentrations yield the compatibility condition

$$
\frac{\left|\int_{0}^{1} W_{1} d z+\tau_{p m 1} \int_{0}^{1} W_{1} h(z) d z+\hat{d}^{2} \int_{0}^{1} W_{m 1} d z_{m}+\tau_{1} \hat{d}^{2} \int_{0}^{1} W_{m 1} h_{m}\left(z_{m}\right) d z_{m}+\tau_{p m 2} \int_{0}^{1} W_{1} d z+\tau_{2} \hat{d}^{2} \int_{0}^{1} W_{m 1} d z_{m}\right|_{0}}{=\hat{T}+\hat{d}^{2}+\tau_{1} \tau_{p m 1}\left(\hat{S}_{1}+\hat{d}^{2}\right)+\tau_{2} \tau_{p m 2}\left(\hat{S}_{2}+\hat{d}^{2}\right)}
$$

By substituting expressions for $\left|W_{1}\right|$ and $\left|W_{m 1}\right|$ in equation (41), we obtain an expression for critical Rayleigh number for different basic salinity profiles in both fluid and porous layers.

\subsection{Linear Salinity Profile:}

In this profile $h(z)=h_{m}\left(z_{m}\right)=1$

The critical Rayleigh number for this model is obtained by substituting (42) in (41) and is found to be

$$
R_{c 1}=\frac{\delta_{7}+\left(R_{s 1} \hat{S}_{1}+R_{s 2} \hat{S}_{2}\right) \delta_{5}+\left(R_{s m 1}+R_{s m 2}\right) \delta_{6}}{\hat{T}\left(\delta_{5}+\frac{\hat{d}^{3} \beta^{2} \delta_{6}}{\kappa}\right)} \mid
$$

where

$$
\begin{aligned}
& \qquad \begin{array}{l}
\delta_{1}=\Delta_{19}+\frac{\Delta_{21}}{2}+\frac{\Delta_{23}}{3}+\frac{\Delta_{25}}{4}+\frac{1}{120}, \quad \delta_{2}=\Delta_{20}+\frac{\Delta_{22}}{2}+\frac{\Delta_{24}}{3}+\frac{\Delta_{26}}{4}, \\
\delta_{3}=\Delta_{27}+\frac{\Delta_{29}}{2}+\Delta_{17}\left(\frac{e^{p}-1}{p}\right)+\Delta_{15}\left(\frac{1-e^{-p}}{p}\right), \\
\delta_{4}=\Delta_{28}+\frac{\Delta_{30}}{2}+\Delta_{18}\left(\frac{e^{p}-1}{p}\right)+\Delta_{16}\left(\frac{1-e^{-p}}{p}\right)-\frac{1}{6}, \quad \delta_{5}=\left(1+\tau_{p m 1}+\tau_{p m 2}\right) \delta_{1}+\hat{d}^{2}\left(1+\tau_{1}+\tau_{2}\right) \delta_{3}, \\
\delta_{6}=\left(1+\tau_{p m 1}+\tau_{p m 2}\right) \delta_{2}+\hat{d}^{2}\left(1+\tau_{1}+\tau_{2}\right) \delta_{4}, \quad \delta_{7}=\hat{T}+\hat{d}^{2}+\tau_{1} \tau_{p m 1}\left(\hat{S}+\hat{d}^{2}\right)+\tau_{2} \tau_{p m 2}\left(\hat{S}_{2}+\hat{d}^{2}\right) .
\end{array} \\
& \Delta_{i}^{\prime s} \text { remains same as earlier. }
\end{aligned}
$$

\subsection{Parabolic salinity profile:}

Following Sparrow et al [18], $h(z)=2 z, \quad h_{m}\left(z_{m}\right)=2 z_{m} \mid$

The critical Rayleigh number for this model is obtained by substituting (43) in (41) and is found to be

$$
R_{c 2}=\frac{\delta_{1}+\left(R_{s 1} \hat{S}_{1}+R_{s 2} \hat{S}_{2}\right) A_{2}+\left(R_{s m 1}+R_{s m 2}\right) A_{3}}{\hat{T}\left(A_{2}+\frac{\hat{d}^{3} \beta^{2} A_{3}}{\kappa}\right)}
$$

where 


$$
\begin{aligned}
& \left|\begin{array}{l}
A_{2}=\Delta_{19} \delta_{2}+\Delta_{21} \delta_{3}+\Delta_{23} \delta_{4}+\Delta_{25} \delta_{5}+\delta_{6}+\Delta_{27} \delta_{7}+\Delta_{29} \delta_{8}+\Delta_{17} \delta_{9}+\Delta_{15} \delta_{10} \\
A_{3}=\Delta_{20} \delta_{2}+\Delta_{22} \delta_{3}+\Delta_{24} \delta_{4}+\Delta_{26} \delta_{5}+\Delta_{28} \delta_{7}+\Delta_{30} \delta_{8}+\Delta_{19} \delta_{9}-\delta_{11}
\end{array}\right| \\
& \delta_{1}=\hat{T}+\hat{d}^{2}+\tau_{1} \tau_{p m 1}\left(\hat{S}+\hat{d}^{2}\right)+\tau_{2} \tau_{p m 2}\left(\hat{S}_{2}+\hat{d}^{2}\right) \\
& \delta_{2}=1+\tau_{p m 1}+\tau_{p m 2}, \quad \delta_{3}=\frac{2 \tau_{p m 1}}{3}+\frac{1+\tau_{p m 2}}{2}, \quad \delta_{4}=\frac{\tau_{p m 1}}{2}+\frac{1+\tau_{p m 2}}{3}, \quad \delta_{5}=\frac{2 \tau_{p m 1}}{5}+\frac{1+\tau_{p m 2}}{4}, \\
& \delta_{6}=\frac{\tau_{p m 1}}{72}+\frac{1+\tau_{p m 2}}{120}, \quad \delta_{7}=\hat{d}^{2}\left(1+\tau_{1}+\tau_{2}\right), \quad \delta_{8}=\hat{d}^{2}\left(\frac{2 \tau_{1}}{3}+\frac{1+\tau_{2}}{2}\right), \\
& \delta_{9}=\hat{d}^{2}\left(2 \tau_{1}\left(\frac{e^{p}}{p}-\frac{e^{p}}{p^{2}}+\frac{1}{p^{2}}\right)+\left(1+\tau_{2}\right) \frac{\left(e^{p}-1\right)}{p}\right), \\
& \delta_{10}=\hat{d}^{2}\left(2 \tau_{1}\left(\frac{e^{-p}}{-p}-\frac{e^{-p}}{p^{2}}+\frac{1}{p^{2}}\right)+\left(1+\tau_{2}\right) \frac{\left(1-e^{-p}\right)}{p}\right), \quad \delta_{11}=\hat{d}^{2}\left(\frac{\tau_{1}}{4}+\frac{1+\tau_{2}}{6}\right)
\end{aligned}
$$

\begin{tabular}{|l|l}
$\Delta_{i}^{\prime} s$ & remains same as earlier.
\end{tabular}

\subsection{Inverted Parabolic salinity profile:}

For this case $h(z)=2(1-z), \quad h_{m}\left(z_{m}\right)=2\left(1-z_{m}\right)$

The critical Rayleigh number for this model is obtained by substituting (44) in (41) and is found to be $R_{c 3}=\frac{\delta_{1}+\left(R_{s 1} \hat{S}_{1}+R_{s 2} \hat{S}_{2}\right) A_{2}+\left(R_{s m 1}+R_{s m 2}\right) A_{3}}{\hat{T}\left(A_{2}+\frac{\hat{d}^{3} \beta^{2} A_{3}}{\kappa}\right)}$

$$
\begin{aligned}
& \text { Where } \\
& \begin{array}{l}
A_{2}=\Delta_{19} \delta_{2}+\Delta_{21} \delta_{3}+\Delta_{23} \delta_{4}+\Delta_{25} \delta_{5}+\delta_{6}+\Delta_{27} \delta_{7}+\Delta_{29} \delta_{8}+\Delta_{17} \delta_{9}+\Delta_{15} \delta_{10} \\
A_{3}=\Delta_{20} \delta_{2}+\Delta_{22} \delta_{3}+\Delta_{24} \delta_{4}+\Delta_{26} \delta_{5}+\Delta_{28} \delta_{7}+\Delta_{30} \delta_{8}+\Delta_{19} \delta_{9}-\delta_{11}
\end{array} \\
& \hline \begin{array}{l}
\delta_{1}=\hat{T}+\hat{d}^{2}+\tau_{1} \tau_{p m 1}\left(\hat{S}+\hat{d}^{2}\right)+\tau_{2} \tau_{p m 2}\left(\hat{S}_{2}+\hat{d}^{2}\right) \\
\delta_{2}=1+\tau_{p m 1}+\tau_{p m 2}, \quad \delta_{3}=\frac{\tau_{p m 1}}{3}+\frac{1+\tau_{p m 2}}{2}, \quad \delta_{4}=\frac{\tau_{p m 1}}{6}+\frac{1+\tau_{p m 2}}{3}, \quad \delta_{5}=\frac{\tau_{p m 1}}{60}+\frac{1+\tau_{p m 2}}{4}, \\
\delta_{6}=\tau_{p m 1}\left(\frac{1}{120}-\frac{1}{144}\right)+\frac{1+\tau_{p m 2}}{120}, \quad \delta_{7}=\hat{d}^{2}\left(1+\tau_{1}+\tau_{2}\right), \quad \delta_{8}=\hat{d}^{2}\left(\frac{\tau_{1}}{3}+\frac{1+\tau_{2}}{2}\right), \\
\delta_{9}=\hat{d}^{2}\left(2 \tau_{1}\left(\frac{e^{p}-1}{p^{2}}+\frac{1}{p}\right)+\left(1+\tau_{2}\right) \frac{\left(e^{p}-1\right)}{p}\right), \\
\delta_{10}=\hat{d}^{2}\left(2 \tau_{1}\left(\frac{e^{-p}-1}{p^{2}}+\frac{1}{p}\right)+\left(1+\tau_{2}\right) \frac{\left(1-e^{-p}\right)}{p}\right), \quad \delta_{11}=\hat{d}^{2}\left(\frac{\tau_{1}}{12}+\frac{1+\tau_{2}}{6}\right)
\end{array}
\end{aligned}
$$

\begin{tabular}{|l|l}
\hline$\Delta_{i}^{\prime}$ & remains same as earlier.
\end{tabular} 


\subsection{Piecewise linear Salting below Salinity profile:}

For this case following Currie [3], $h(z)=\left\{\begin{array}{cc}\varepsilon^{-1}, & 0 \leq z \leq \varepsilon \\ 0, & \varepsilon \leq z \leq 1\end{array}, h_{m}\left(z_{m}\right)=\left\{\begin{array}{cc}\varepsilon_{m}^{-1}, & 0 \leq z_{m} \leq \varepsilon_{m} \\ 0, & \varepsilon_{m} \leq z_{m} \leq 1\end{array}\right.\right.$

The critical Rayleigh number for this model is obtained by substituting (45) in (41) and is found to be

$$
R_{c 4}=\frac{\delta_{1}+\left(R_{s 1} \hat{S}_{1}+R_{s 2} \hat{S}_{2}\right) A_{2}+\left(R_{s m 1}+R_{s m 2}\right) A_{3}}{\hat{T}\left(A_{2}+\frac{\hat{d}^{3} \beta^{2} A_{3}}{\kappa}\right)}
$$

where

$$
\begin{aligned}
& \mid \begin{array}{l}
A_{2}=\Delta_{19} \delta_{2}+\Delta_{21} \delta_{3}+\Delta_{23} \delta_{4}+\Delta_{25} \delta_{5}+\delta_{6}+\Delta_{27} \delta_{7}+\Delta_{29} \delta_{8}+\Delta_{17} \delta_{9}+\Delta_{15} \delta_{10}, \\
A_{3}=\Delta_{20} \delta_{2}+\Delta_{22} \delta_{3}+\Delta_{24} \delta_{4}+\Delta_{26} \delta_{5}+\Delta_{28} \delta_{7}+\Delta_{30} \delta_{8}+\Delta_{19} \delta_{9}-\delta_{11}, \\
\delta_{1}=\hat{T}+\hat{d}^{2}+\tau_{1} \tau_{p m 1}\left(\hat{S}+\hat{d}^{2}\right)+\tau_{2} \tau_{p m 2}\left(\hat{S}_{2}+\hat{d}^{2}\right), \quad \delta_{2}=1+\tau_{p m 1}+\tau_{p m 2}, \quad \delta_{3}=\frac{1}{2}\left(\varepsilon \tau_{p m 1}+1+\tau_{p m 2}\right), \\
\delta_{4}=\frac{1}{3}\left(\varepsilon^{2} \tau_{p m 1}+1+\tau_{p m 2}\right), \quad \delta_{5}=\frac{1}{4}\left(\varepsilon^{3} \tau_{p m 1}+1+\tau_{p m 2}\right), \quad \delta_{6}=\frac{1}{120}\left(\varepsilon^{4} \tau_{p m 1}+1+\tau_{p m 2}\right), \\
\hline \delta_{7}=\hat{d}^{2}\left(1+\tau_{1}+\tau_{2}\right), \quad \delta_{8}=\frac{\hat{d}^{2}}{2}\left(\varepsilon \tau_{1}+1+\tau_{2}\right), \quad \delta_{9}=\frac{\hat{d}^{2}}{p}\left(\frac{\tau_{1}}{\varepsilon_{m}}\left(e^{p \varepsilon_{m}-1}\right)+\left(1+\tau_{2}\right)\left(e^{p}-1\right)\right), \\
\delta_{10}=\frac{\hat{d}^{2}}{p}\left(\frac{\tau_{1}}{\varepsilon_{m}}\left(1-e^{-p \varepsilon_{m}}\right)+\left(1+\tau_{2}\right)\left(1-e^{-p}\right)\right), \quad \delta_{11}=\hat{d}^{2}\left(\frac{\tau_{1} \varepsilon_{m}^{2}}{2}+\frac{\left(1+\tau_{2}\right)}{6}\right)
\end{array} \\
& \Delta_{i}^{\prime s} \text { are defined earlier. }
\end{aligned}
$$

\subsection{Piecewise linear Salinity profile Desalting above:}

For this case following Vidal and Acrivos [21],

$h(z)=\left\{\begin{array}{cc}0, & 0 \leq z \leq(1-\varepsilon) \\ \varepsilon^{-1}, & (1-\varepsilon) \leq z \leq 1\end{array} \quad, h_{m}\left(z_{m}\right)=\left\{\begin{array}{cc}0, & 0 \leq z_{m} \leq\left(1-\varepsilon_{m}\right) \\ \varepsilon_{m}^{-1}, & \left(1-\varepsilon_{m}\right) \leq z_{m} \leq 1\end{array}\right.\right.$

The critical Rayleigh number for this model is obtained by substituting (46) in (41) and is found to be $R_{c 5}=\frac{\delta_{1}+\left(R_{s 1} \hat{S}_{1}+R_{s 2} \hat{S}_{2}\right) A_{2}+\left(R_{s m 1}+R_{s m 2}\right) A_{3}}{\hat{T}\left(A_{2}+\frac{\hat{d}^{3} \beta^{2} A_{3}}{\kappa}\right)}$

where

$A_{2}=\Delta_{19} \delta_{2}+\Delta_{21} \delta_{3}+\Delta_{23} \delta_{4}+\Delta_{25} \delta_{5}+\delta_{6}+\Delta_{27} \delta_{7}+\Delta_{29} \delta_{8}+\Delta_{17} \delta_{9}+\Delta_{15} \delta_{10}$
$A_{3}=\Delta_{20} \delta_{2}+\Delta_{22} \delta_{3}+\Delta_{24} \delta_{4}+\Delta_{26} \delta_{5}+\Delta_{28} \delta_{7}+\Delta_{30} \delta_{8}+\Delta_{19} \delta_{9}-\delta_{11}$




$$
\left\{\begin{array}{l}
\delta_{1}=\hat{T}+\hat{d}^{2}+\tau_{1} \tau_{p m 1}\left(\hat{S}+\hat{d}^{2}\right)+\tau_{2} \tau_{p m 2}\left(\hat{S}_{2}+\hat{d}^{2}\right), \quad \delta_{2}=1+\tau_{p m 1}+\tau_{p m 2}, \\
\delta_{3}=\frac{1}{2}\left(\frac{\tau_{p m 1}}{\varepsilon}\left(1-(1-\varepsilon)^{2}\right)+\left(1+\tau_{p m 2}\right)\right), \quad \delta_{4}=\frac{1}{3}\left(\frac{\tau_{p m 1}}{\varepsilon}\left(1-(1-\varepsilon)^{3}\right)+\left(1+\tau_{p m 2}\right)\right), \\
\delta_{5}=\frac{1}{4}\left(\frac{\tau_{p m 1}}{\varepsilon}\left(1-(1-\varepsilon)^{4}\right)+\left(1+\tau_{p m 2}\right)\right), \quad \delta_{6}=\frac{1}{120}\left(\frac{\tau_{p m 1}}{\varepsilon}\left(1-(1-\varepsilon)^{5}\right)+\left(1+\tau_{p m 2}\right)\right), \\
\delta_{7}=\hat{d}^{2}\left(1+\tau_{1}+\tau_{2}\right), \quad \delta_{8}=\frac{\hat{d}^{2}}{2}\left(\frac{\tau_{1}}{\varepsilon_{m}}\left(1-\left(1-\varepsilon_{m}\right)^{2}\right)+\left(1+\tau_{2}\right)\right), \\
\delta_{9}=\frac{\hat{d}^{2}}{p}\left(\frac{\tau_{1}}{\varepsilon_{m}}\left(e^{p}-e^{p\left(1-\varepsilon_{m}\right)}\right)+\left(1+\tau_{2}\right)\left(e^{p}-1\right)\right), \quad \delta_{10}=\frac{\hat{d}^{2}}{p}\left(\frac{\tau_{1}}{\varepsilon_{m}}\left(-e^{-p}+e^{-p\left(1-\varepsilon_{m}\right)}\right)+\left(1+\tau_{2}\right)\left(1-e^{-p}\right)\right) \\
\delta_{11}=\frac{\hat{d}^{2}}{6}\left(\tau_{1}\left(\left(1-\varepsilon_{m}\right)^{3}-1\right)+\left(1+\tau_{2}\right)\right) .
\end{array}\right.
$$

are defined earlier.

\subsection{Step function salinity profile:}

In this profile the basic concentration/solute/salt drops suddenly by an amount $\Delta S$ at $z=\varepsilon$ and $\Delta S_{m}$ a $z_{m}=\varepsilon_{m}$ otherwise uniform. Accordingly, $h(z)=\delta(z-\varepsilon), \quad h_{m}\left(z_{m}\right)=\delta\left(z_{m}-\varepsilon_{m}\right)$

where $\varepsilon$ is the solutal depth in the fluid layer and $\varepsilon_{m}$ is the solutal depth in the porous layer.

The critical Rayleigh number for this model is obtained by substituting (47) in (41) and is found to be

$$
R_{c 6}=\frac{\delta_{1}+\left(R_{s 1} \hat{S}_{1}+R_{s 2} \hat{S}_{2}\right) A_{2}+\left(R_{s m 1}+R_{s m 2}\right) A_{3}}{\hat{T}\left(A_{2}+\frac{\hat{d}^{3} \beta^{2} A_{3}}{\kappa}\right)}
$$

where

$$
\begin{aligned}
& \left|\begin{array}{l}
A_{2}=\Delta_{19} \delta_{2}+\Delta_{21} \delta_{3}+\Delta_{23} \delta_{4}+\Delta_{25} \delta_{5}+\delta_{6}+\Delta_{27} \delta_{7}+\Delta_{29} \delta_{8}+\Delta_{17} \delta_{9}+\Delta_{15} \delta_{10} \\
A_{3}=\Delta_{20} \delta_{2}+\Delta_{22} \delta_{3}+\Delta_{24} \delta_{4}+\Delta_{26} \delta_{5}+\Delta_{28} \delta_{7}+\Delta_{30} \delta_{8}+\Delta_{19} \delta_{9}-\delta_{11}
\end{array}\right| \\
& \delta_{1}=\hat{T}+\hat{d}^{2}+\tau_{1} \tau_{p m 1}\left(\hat{S}+\hat{d}^{2}\right)+\tau_{2} \tau_{p m 2}\left(\hat{S}_{2}+\hat{d}^{2}\right), \quad \delta_{2}=1+\tau_{p m 1}+\tau_{p m 2}, \quad \delta_{3}=\varepsilon \tau_{p m 1}+\frac{1+\tau_{p m 2}}{2} \text {, } \\
& \delta_{4}=\varepsilon^{2} \tau_{p m 1}+\frac{1+\tau_{p m 2}}{3}, \quad \delta_{5}=\varepsilon^{3} \tau_{p m 1}+\frac{1+\tau_{p m 2}}{4}, \quad \delta_{6}=\frac{\varepsilon^{4} \tau_{p m 1}}{24}+\frac{1+\tau_{p m 2}}{120}, \quad \delta_{7}=\hat{d}^{2}\left(1+\tau_{1}+\tau_{2}\right), \\
& \delta_{8}=\hat{d}^{2}\left(\varepsilon_{m} \tau_{1}+\frac{1+\tau_{2}}{2}\right), \quad \delta_{9}=\hat{d}^{2}\left(\tau_{1} e^{p \varepsilon_{m}}+\left(1+\tau_{2}\right) \frac{\left(e^{p}-1\right)}{p}\right) \text {, } \\
& \delta_{10}=\hat{d}^{2}\left(\tau_{1} e^{-p \varepsilon_{m}}+\left(1+\tau_{2}\right) \frac{\left(1-e^{-p}\right)}{p}\right), \quad \delta_{11}=\hat{d}^{2}\left(\frac{\tau_{1} \varepsilon_{m}^{2}}{2}+\frac{\left(1+\tau_{2}\right)}{6}\right) .
\end{aligned}
$$

$\Delta_{i}^{s}$ are defined earlier. 


\section{RESULTS AND DISCUSSIONS}

For Linear, Parabolic and Inverted Parabolic Salinity Profiles:

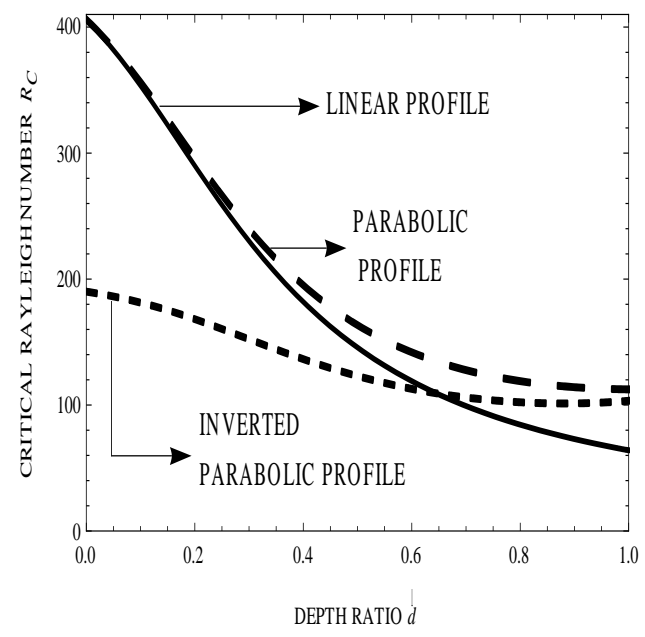

Fig.1. The variation of critical thermal Rayleigh number $\left|R_{c}\right|$ for Linear, Parabolic and Inverted parabolic salinity profiles with respect to the depth ratio $\hat{d}=\frac{d_{m}}{d}$.

Figure1 shows the variation of critical Rayleigh number $R_{c}$ for different profiles with respect to the depth ratio for fixed values of $D a=0.1, \quad \kappa=1, \quad \mu=2, \quad \tau_{1}=\tau_{2}=0.25, \quad \tau_{p m 1}=\tau_{p m 2}=0.75, \quad \hat{S}_{1}=\hat{S}_{2}=1, R_{s 1}=R_{s 2}=5$ and $\hat{T}=1$. Graphically it is evident that the parabolic salinity profile is the most stable. Inverted parabolic profile is unstable for $0 \leq \hat{d} \leq 0.65$ and linear profile is unstable for $0.65 \leq \hat{d} \leq 1$. At $\hat{d}=0.65$ linear and inverted parabolic profiles have same effect on $R_{c}$.
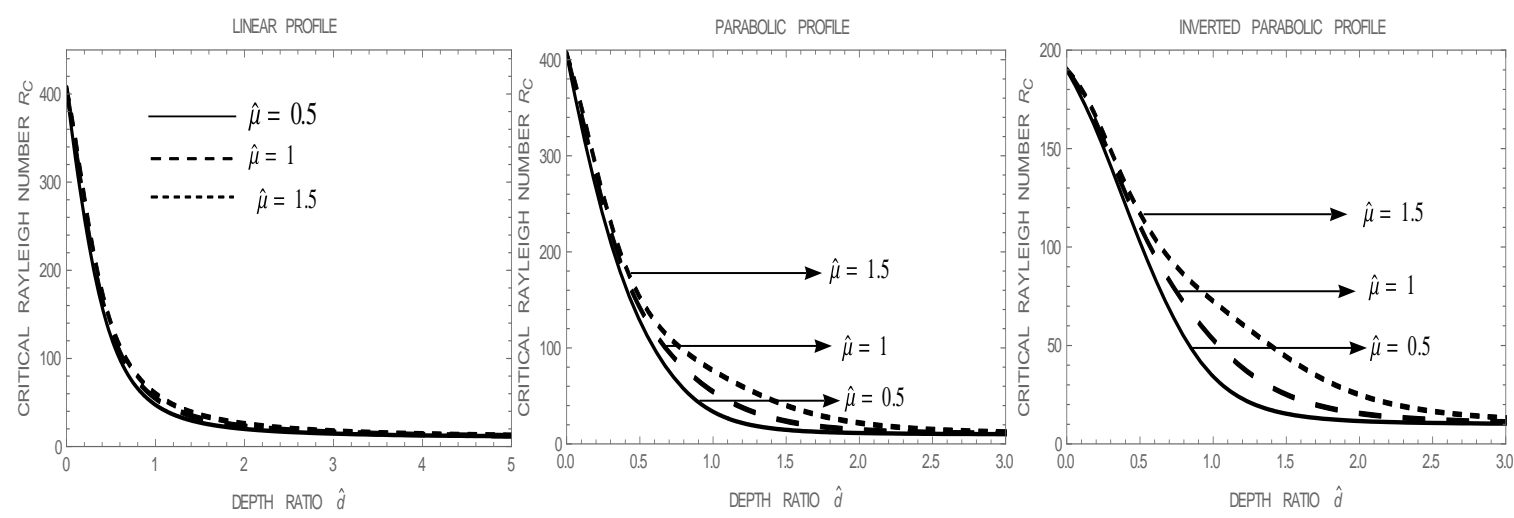

Fig.2: The effect of $\hat{\mu}$ on critical Rayleigh number $R_{c} \mid$ for Linear, Parabolic and Inverted parabolic profiles with respect to the depth ratio $\hat{d}=\frac{d_{m}}{d}$.

Figure 2 shows the variation of critical Rayleigh number $\left.\mid R_{c}\right\rfloor$ for different profiles with respect to the depth ratio for fixed values of $D a=0.1, \quad \kappa=1, \quad \tau_{1}=\tau_{2}=0.25, \quad \tau_{p m 1}=\tau_{p m 2}=0.75, \quad R_{s 1}=R_{s 2}=5, \quad \hat{S}_{1}=\hat{S}_{2}=1$, and $\hat{T}_{1}=1$. The effects of the viscosity ratio $\hat{\mu}=\mu_{m} / \mu$ which is the ratio of the effective viscosity of the porous matrix to that of the fluid viscosity is displayed in the above graphs. For fixed values of depth ratio, the increase in the value of $\hat{\mu}$ increases the value of critical Rayleigh number $\left\lfloor R_{c} \mid\right.$ i.e., the system is stabilized. Thus the onset of triple diffusive convection is delayed. 

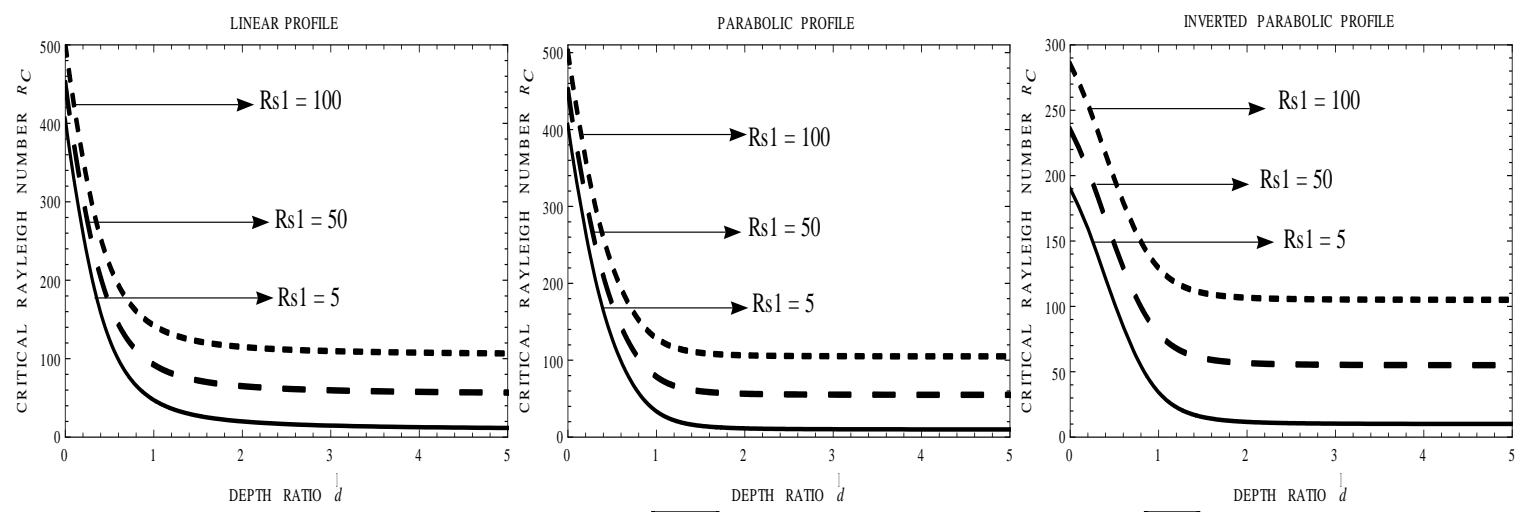

Fig.3. The effect of first solute Rayleigh number $R_{s 1}$ on critical Rayleigh number $R_{c}$ for Linear, Parabolic and Inverted parabolic profiles with respect to the depth ratio $\hat{d}=\frac{d_{m}}{d}$. Figure 3 shows the variation of critical Rayleigh number $R_{c}$ for different profiles with respect to the depth ratio for fixed values of

$$
D a=0.1, \kappa=1, \mu=0.5, \tau_{1}=\tau_{2}=0.25, \tau_{p m 1}=\tau_{p m 2}=0.75, R_{s 2}=5, \hat{S}_{1}=\hat{S}_{2}=1, \text { and } \quad \hat{T}=1 . \text { The }
$$
effect of solute Rayleigh number of first solute $R_{s 1}=\frac{g \alpha_{s 1}\left(C_{10}-C_{1 u}\right) d^{3}}{v \kappa}$ is displayed in the above graphs. As the curves are diverging the effect of Solute Rayleigh number $R_{s 1}$ is large for small change in the value of depth ratio. From the curves it is evident that for fixed values of depth ratio, the increase in the value of solute Rayleigh number $R_{s 1}$ increases the value of critical Rayleigh number $R_{c} \mid$ i.e., the system is stabilized. Thus the onset of triple diffusive convection is delayed. The increasing values of solute Rayleigh number $R_{s 1}$ will affect the onset of convection only for larger values of the depth ratio $\hat{d}=\frac{d_{m}}{d}$ that is, in porous layer dominant composite systems the convection is delayed.
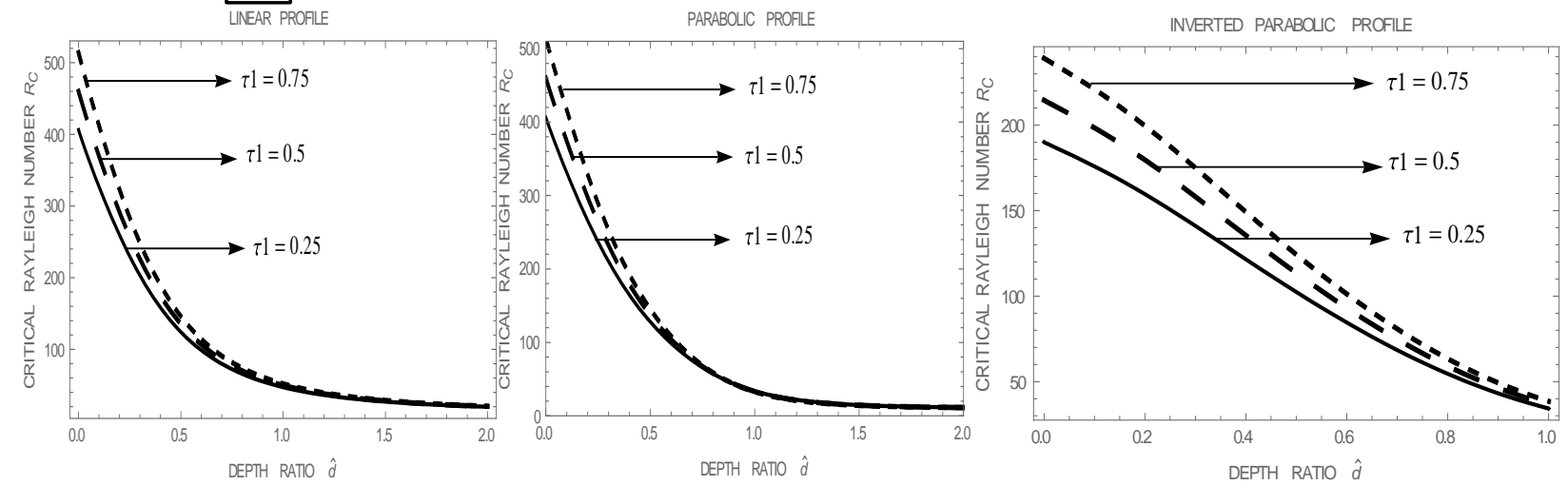

Fig.4 The effect of $\tau_{1}$ on critical Rayleigh number $R_{c}$ for Linear, Parabolic and Inverted parabolic profiles with respect to the depth ratio $\hat{d}=\frac{d_{m}}{d}$.

Figure 4 shows the effects of the diffusivity ratiq $\tau_{1}=\frac{\kappa_{1}}{\kappa} \mid$, which is the ratio of first saline diffusivity to thermal diffusivity of the fluid on critical Rayleigh number $R_{c}$ for different profiles with respect to the depth ratio for fixed values $\quad$ of $D a=0.1, \kappa=1, \mu=0.5$,
$\tau_{2}=0.25, \tau_{p m 1}=\tau_{p m 2}=0.75, R_{s 1}=R_{s 2}=50$, and
$\hat{S}_{1}=\hat{S}_{2}=1, \hat{T}=1$. It is clear from the graphs that all the three curves are converging which shows that for larger values of the depth rati $\left\lfloor\hat{d}=\frac{d_{m}}{d} \mid\right.$, there is no effect of any variation in the values of $\tau_{1}$. The effect of $\tau_{1}$ is prominent for fluid layer dominant composite systems. For a fixed value of depth ratio, the increase in the value of $\tau_{1}$ increases the value of the critical thermal Rayleigh number. Thus increasing values of $\tau_{1}$ makes the system stable and hence delay the convection. 
For Salting below, Desalting above and Step function Salinity Profiles:

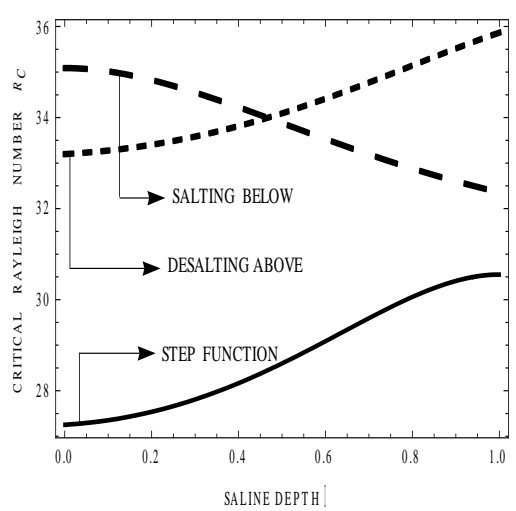

Fig.5: The variation of critical Rayleigh number $\left\lfloor R_{c}\right\rfloor$ for Step function, Desalting above and Salting below profiles with respect to the saline depth $\varepsilon$.

Figure 5 shows the variation of critical Rayleigh number $\left|R_{c}\right|$ for different profiles with respect to the saline depth $\varepsilon$ for fixed $\quad$ values $\quad D a=0.1, \hat{\mu}=0.5, \quad \hat{d}=1, \quad \varepsilon_{m}=1, \quad \kappa=1, \hat{S}_{1}=\hat{S}_{2}=1, \hat{T}=1, \quad R_{s 1}=R_{s 2}=5$, $\tau_{1}=\tau_{2}=0.25, \tau_{p m 1}=\tau_{p m 2}=0.75$.

Graphically it is evident that the step function salinity profile is the unstable profile. Salting below salinity profile is the stable profile for the depth ratio $0 \leq \hat{d} \leq 0.45$ and Desalting Above salinity profile is the stable profile for the depth ratio $0.45 \leq \hat{d} \leq 1$. At $\hat{d}=0.45$ both salting below and desalting above profiles have same effect on $R_{c}$.
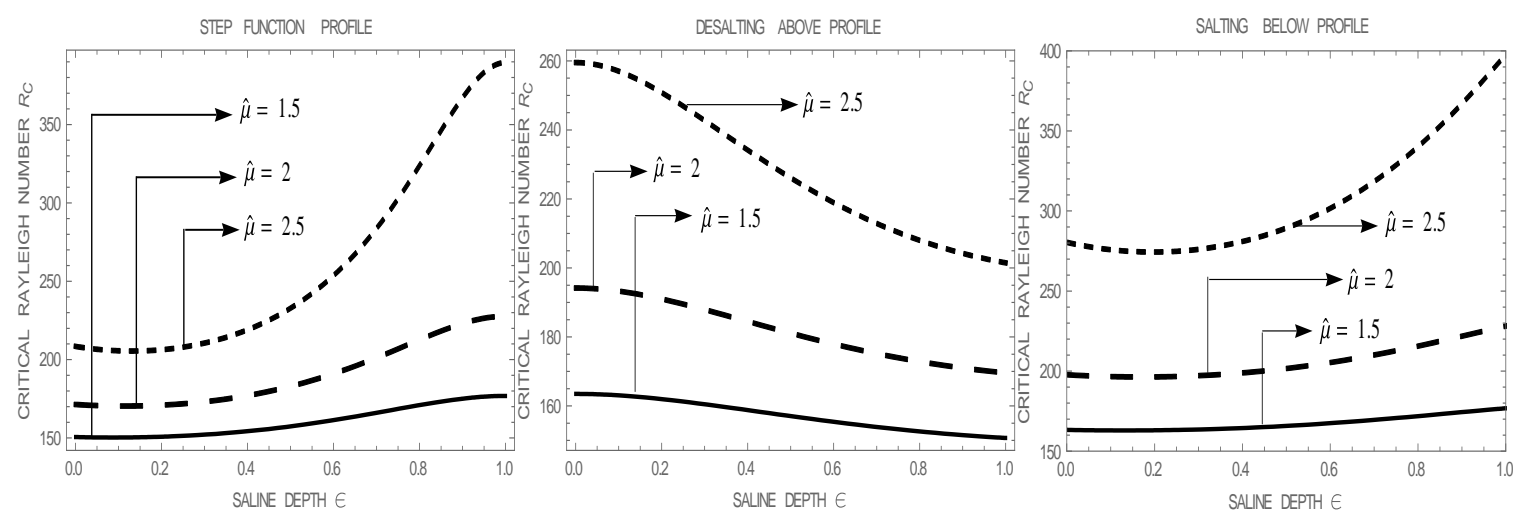

Fig.6: The effect of $\hat{\mu}$ on critical Rayleigh number $R_{c}$ for Step function, Desalting above and Salting below profiles with respect to the saline $\operatorname{depth} \varepsilon$.

Figure 6 shows the effects of the viscosity ratio $\hat{\mu}=\frac{\mu_{m}}{\mu}=1.5,2,2.5$, which is the ratio of the effective

viscosity of the porous matrix to that of the fluid layer on critical Rayleigh number $R_{c}$. For fixed value of $D a=0.1, \quad \hat{d}=1, \quad \varepsilon_{m}=1, \quad \kappa=1, \hat{S}_{1}=\hat{S}_{2}=1, \quad \hat{T}=1, \quad R_{s 1}=R_{s 2}=5, \tau_{1}=\tau_{2}=0.25, \tau_{p m 1}=\tau_{p m 2}=0.75$. With the increase in the value of $|\hat{\mu}|$ increases the critical thermal Rayleigh $R_{c}$ which stabilizes the system, so the $\mu_{m}$ is made larger than the fluid viscosity $\mu$, the onset of the convection in the fluid layer can be delayed.

onset of triple diffusive convection is delayed. In other words, when the effective viscosity of the porous medium 


\section{Non - Darcian and Non - Uniform Salinity Gradients on Triple Diffusive Convection in Composite Layers}
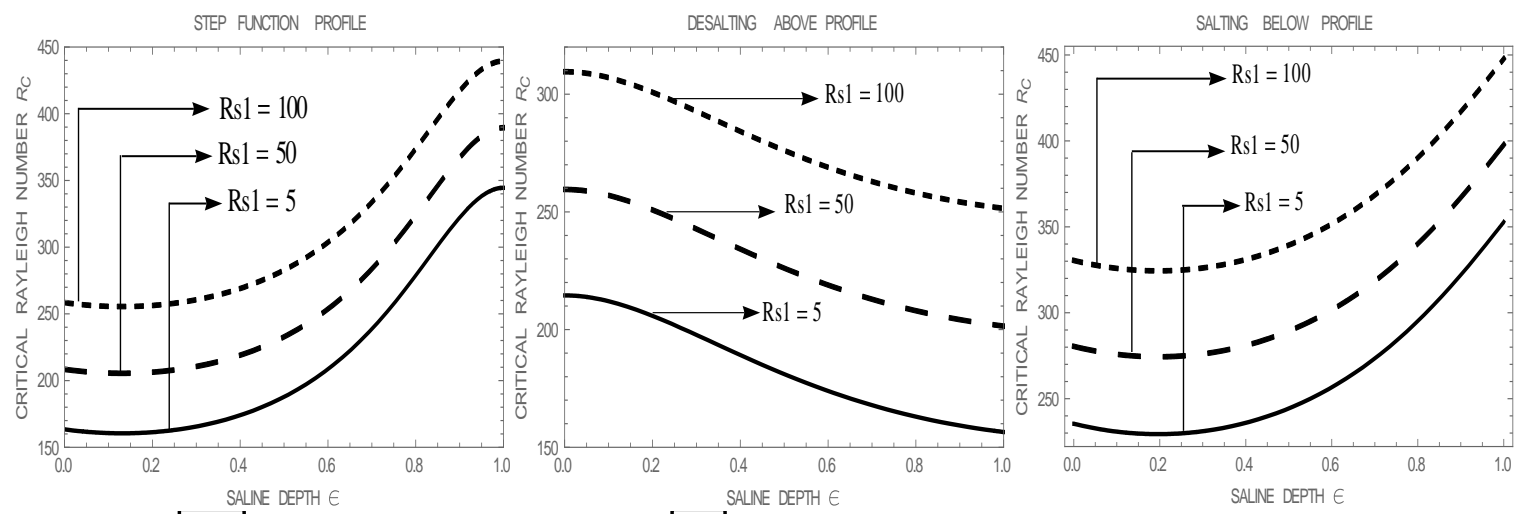

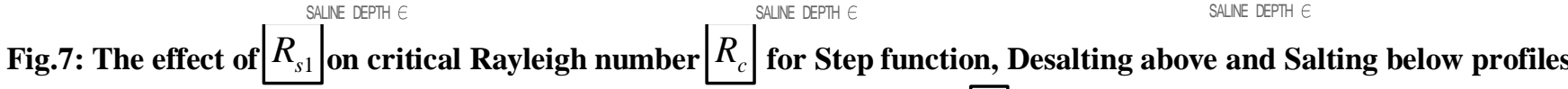
with respect to the saline depth $\varepsilon$.

Figure 7 shows the effect of solute Rayleigh number of first solut $R_{s 1}=\frac{g \alpha_{s 1}\left(C_{10}-C_{1 u}\right) d^{3}}{v \kappa}=5,50,100$. For fixed values of $\quad D a=0.1, \quad \hat{d}=1, \quad \varepsilon_{m}=1, \quad \kappa=1, \quad \hat{\mu}=0.5, \quad \hat{S}_{1}=\hat{S}_{2}=1, \quad \hat{T}=1, \quad R_{s 2}=5, \tau_{1}=\tau_{2}=0.25$, $\tau_{p m 1}=\tau_{p m 2}=0.75$.

From the above graphs it is evident that for fixed values of saline depth ' $\varepsilon$ ' the increase in the value of solute Rayleigh number $R_{s 1}$ increases the value of critical

Rayleigh number $\left.\mid R_{c}\right\rfloor$ i.e., the system is stabilized. Thus the onset of triple diffusive convection is delayed.
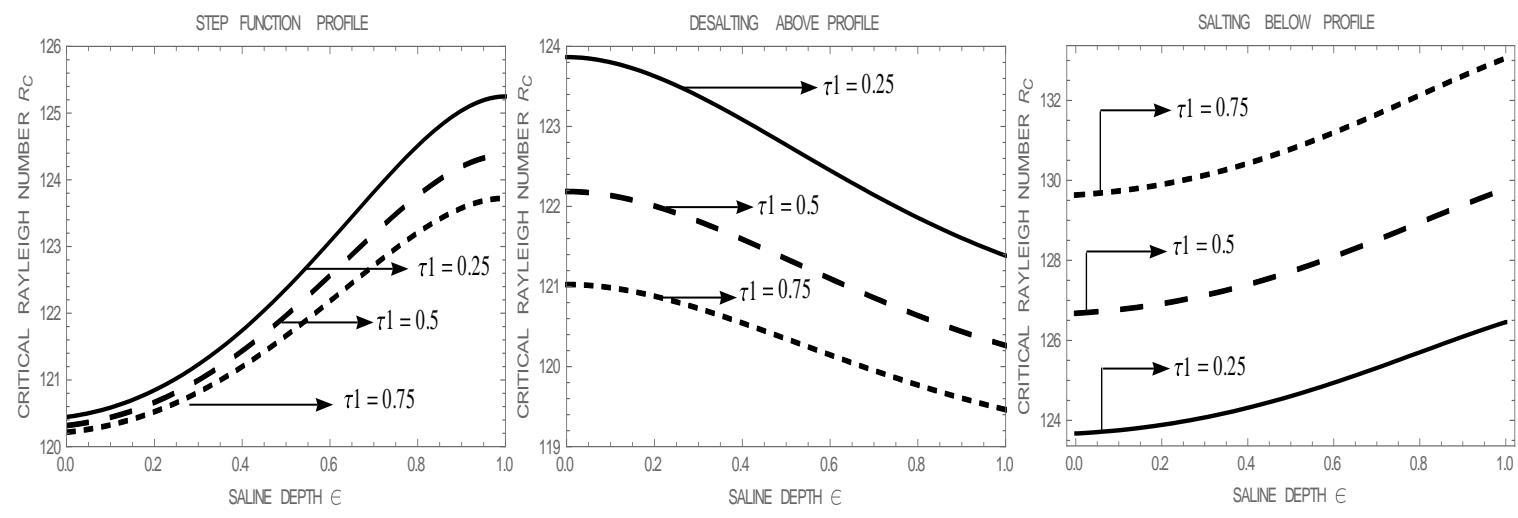

Figure 8. The effect of $\left|\tau_{1}\right|$ on critical Rayleigh number $\left|R_{c}\right|$ for Step function, Desalting above and Salting below profiles with respect to the saline depth $\varepsilon$.

Figure 8 shows the effects of the diffusivity ratio $\tau_{1}=\frac{\kappa_{1}}{\kappa}=0.25,0.5,0.75$, which is the ratio of first saline to thermal diffusivity of the fluid for fixed values

\begin{tabular}{ll}
$D a=0.1, \quad \hat{\mu}=0.5, \quad \hat{d}=1, \quad \varepsilon_{m}=1, \quad \kappa=1$, & $\hat{S}_{1}=\hat{S}_{2}=1, \hat{T}=1, \quad R_{s 1}=R_{s 2}=5$, \\
\hline$\tau_{2}=0.25, \tau_{p m 1}=\tau_{p m 2}=0.75$.
\end{tabular}

From the graph it is clear that critical Rayleigh number $R_{c}$ decreases as $\tau_{1}$ increases in step function and desalting above profiles and $R_{c}$ increases with increase the in $\tau_{1}$ in Salting below profile. Thus the system is destabilized for step function and desalting above profile and stabilized for Salting below profile. 


\section{CONCLUSION:}

6.1. For Linear, Parabolic and Inverted Parabolic salinity Profile:

i) The curves of solute Rayleigh number of first solute $R_{s 1}$ are diverging, indicating that, in porous layer dominant composite systems the convection is delayed by increasing solute Rayleigh number $R_{s 1}$.

ii) The curves of diffusivity ratio $\tau_{1}$ are converging, indicating that, in porous layer dominant composite systems the convection can be made fast by increasing the concentration of first salt.

\subsection{For Salting below, Desalting above and Step} function Profile:

i) By increasing the parameters and $\hat{\mu}$ triple diffusive convection for the above profiles is delayed.

ii) By increasing the thermal diffusivity ratid $\tau_{1}$, the triple diffusive convection in the above profiles is quick.

\section{REFERENCES}

1. Chand. S, 2013. Triple-diffusive convection in a micropolar ferrofluid in the presence of rotation. Int. J. of Applied Mechanics and Engineering, 18, 307-327.

2. Chen F and Chen C F, 1988. Onset of Finger Convection in a horizontal porous layer underlying a fluid layer, J. Heat transfer, $110,403$.

3. Currie I G, 1967. Isotropic composition and origin of the Red sea and Salton Sea geothermal brines, Science, 154, 1544.

4. Griffiths R.W, 1979. The Influence of a third Diffusing Component upon the onset of Convection, J. Fluid Mech. vol. 92,659 .

5. Lopez A.R, Romero L.A and Pearlstein A.J, 1990. Effect of rigid boundaries on the onset of Convective Instability in a Triply Diffusive Fluid Layer. Physics of Fluids, 2, 897.

6. Mukesh Kumar Awasthi, Vivek Kumar and Ravi Kumar Patel, 2018. Onset of triply diffusive convection in a Maxwell fluid saturated porous layer with internal heat source, Engineering Physics and Mathematics, 9, 1591-1600.

7. Nield D A, 1977. Onset of convection in a fluid layer overlying a layer of a porous medium, J. Fluid Mech., 81,513.

8. Pearlstein A.J, Harris R.M and Terrones, 1989. The onset of Convective Instability in a Triply Diffusive Fluid Layer, .J. Fluid Mech., 202, 443.

9. Raghunatha K.R, Shivakumara I S and B. M. Shankar, 2018, Weakly nonlinear stability analysis of triple diffusive convection in a Maxwell fluid saturated porous layer, Applied Mathematics and Mechanics, 39, 153 - 168.

10. Raghunatha K.R and Shivakumara I.S, 2018. Stability of triple diffusive convection in a viscoelastic fluid saturated porous layer. Applied Mathematics and Mechanics, 39, 1385-1410.

11. Rana G C, Ramesh Chand, Veena Sharma and Abhilasha Sharda, 2016. On the onset of triple-diffusive convection in a layer of nanofluid, JCAMECH, 47, 67-77.

12. Rionero S, 2013a. Triple Diffusive Convection in Porous Media, Act Mech. 224, 447.

13. Rionero S, 2013b. Multicomponent Diffusive Convective Fluid motions in Porous Layers ultimately boundedness, absence of subcritical Instability, and global nonlinear stability for any number of salts. Phys Fluids, 25, 1 .

14. Sameena Tarannum and S. Pranesh, 2017. Heat and Mass Transfer of Triple Diffusive Convection in a Rotating Couple Stress Liquid using Ginzburg-Landau Model, International Journal of Mechanical and Mechatronics Engineering, 11, 3.

15. Shivakumara I.S, Suma and Krishna B, 2006. Onset of surface tension driven convection in superposed layers of fluid and saturated porous medium, Arch. Mech., 58, 71-92.
16. Shivakumara I.S and Kumar S.B.N, 2013. Bifurcation in Triply Diffusive Couple Stress Fluid Systems. International Journal of Engineering Research and Applications, 3, 372.

17. Shivakumara.I.S, Kumar S.B.N, 2014. Linear and Weakly Nonlinear Triple Diffusive Convection in a Couple Stress Fluid Layer, International Journal of Heat and Mass Transfer, 68, 542.

18. Sparrow E M, Goldstein R J and Jonsson V K, 1964. Thermal instability in a horizontal fluid Lauer: Effect of boundary conditions and nonlinear temperature profile, J. Fluid Mechanics, $18,513$.

19. Turner J.S, 1985. Multicomponent Convection, Ann. Rev. Fluid Mech. 17, 11-44p.

20. Venkatachalappa M, Prasad, Shivakumara I.S and Sumithra R, 1997. Hydrothermal growth due to double diffusive convection in composite materials, Proceedings of 14th National Heat and Mass Transfer Conference and 3rd ISHMT ASME Joint Heat and Mass transfer conference, 29-31.

21. Vidal A and Acrivos A, 1966. Nature of the neutral state in surface tension driven convection, Phys. Fluids, 9, 615.

22. Zhao M, Wang S and Zhang Q, 2013. Onset of Triply Diffusive Convection in a Maxwell Fluid Saturated Porous Layer. Applied Mathematical Modelling. 38, 23-52. 